\title{
Sharing of NKN Facilities in Static Step Topology under Distributed Environment
}

\author{
Taskeen Zaidi, Vipin Saxena
}

Department of Computer Science, Babasaheb Bhimrao Ambedkar University, Lucknow, India.

Email: taskeenzaidi867@gmail.com,vsax1@rediffmail.com

Received March 12 ${ }^{\text {th }}, 2013$; revised April 15 ${ }^{\text {th }}, 2013$; accepted April 23 ${ }^{\text {rd }}, 2013$

Copyright (C 2013 Taskeen Zaidi, Vipin Saxena. This is an open access article distributed under the Creative Commons Attribution License, which permits unrestricted use, distribution, and reproduction in any medium, provided the original work is properly cited.

\begin{abstract}
The government of India has set up distributed network in India for sharing the knowledge among the higher learning Institutions in the year 2009 under Next Generation Services (NGN) and slowly-slowly it is expanding as the distributed computing network becomes very popular now days due to low cost involvement. This network is called as a National Knowledge Network (NKN) whose main purpose is to connect all the Indian universities, research institutions, research labs, digital libraries, countrywide classroom, etc. under one platform. In the present work, sharing of different kinds of facilities under NKN is demonstrated for the static step topology designed by the authors. The different kinds of facilities are connected through step topology and a well known modeling technique i.e. Unified Modeling Language (UML) is used for demonstrating the UML class diagram for sharing of all these facilities through step topology. Some experimental results are also demonstrated for the topology.
\end{abstract}

Keywords: Distributed System; NKN; Step Topology; UML

\section{Introduction}

Recently, it is observed from the literature that the distributed approach of computing is much more popular over the centralized approach. As the benefits of distributed approach that is it takes low cost involvement and execute the tasks in faster manner through synchronization concept. This kind of system does not share the global clock. Wide Area Network (WAN) is the best example of a distributed computing system. On the basis of this approach, high speed network connectivity came into existence in the year 2009 in India and National Knowledge Network (NKN) was established under Next Generation Network (NGN) services. The main aim of NKN is to bring all services like universities, research labs, institutions, E-governance, internet, digital libraries, and country wide classroom under one platform. The NKN is very fast and it supports transfer of resources up to $1 \mathrm{Gbps}$, which can be enhanced up to $20 \mathrm{Gbps}$ in the future. The present paper deals with the impact of NKN network as well as the sharing of facilities connected in static step topology. A UML class diagram is also designed by author for the execution of tasks and allocation of resources under NKN.

Distributed computing approach is very popular now days, due to low cost involvement and easier implementation of its infrastructure and fast transmission speed. It supports various features like data sharing, resources sharing, audio sharing, videos sharing, etc without using global clock. Tanenbaum [1] has written a book on networking in which various types of static and dynamic topologies are described by authors. Forouzan [2] has well described the network criteria, structures and models of networks, as well as devices used in networking. Distributed computing approach used various types of algorithms for execution of tasks under the mutual exclusion condition for allocation of resources; these are well explained by Siberschatz and Galvin [3]. UML profiles for distributed and parallel execution of tasks are well studied by Pllana and Fahringer [4,5]. As UML is a platform independent language, it features and versions are well described by Booch [6,7]. The UML is launched by Object Management Group (OMG), its specifications, versions and features are well explained in [8]. Saxena and Arora [9] have designed a protocol for mutual exclusion under distributed environment for the bidirectional ring. Saxena and Arora [10] have also evaluated the performance for object-oriented software systems. A distributed computing approach was used to set up NKN for connecting all the facilities like universities, research 
institutions, research labs, health-care, E-governance, grid computing, internet, digital library and country wide classroom. The impact of National Knowledge Network as well as facilities provided by this network, device used for implementation of this network, as well as the advantages and disadvantages of NKN are available in [11]. Gloria et al. [12] has compared the traditional approach of learning with e-learning in higher education. Zhang and Bao [13] explained research aspects on computer technology using e-learning in higher education. Zaidi and Saxena [14] have designed a static step topology for interconnection of facilities under distributed environment with varying steps and can be extended into numbers of devices. Zaidi and Saxena [15] have also studied the percentage utilization of NKN network in an Institution and also the impact of NKN is well explained in the paper along with the advantages and disadvantages of NKN. The paper also described the issues and challenges in the new kind of NKN network.

\section{Background}

\subsection{Process}

A process is defined as a subroutine, macro, sub program, subtask that is running on the processors. Process Execution Controller (PEC) is fully responsible to execute the process. UML class diagram for process is represented below in Figure 1.

Process is categorized with its id called as the Process_id and other attributes of a Process are Process_size, Process_in_time, Process_out_time, Process_priority, and methods on Process class are Process_create(), Process_ delete(), Process_update(), Process_join(), Process_suspend() and Process_synchronize() as represented in Figure 1.

\subsection{A Distributed System and NKN}

In India National Knowledge Network (NKN) is an ex-

\begin{tabular}{|c|}
\hline $\begin{array}{c}<\text { processing_unit }>> \\
\text { Process }\end{array}$ \\
\hline $\begin{array}{l}\text { +Process_id: integer } \\
\text { +Process_size: integer } \\
\text { +Process_in_time: string } \\
\text { +Process_out_time: string } \\
\text { +Process_priority: integer }\end{array}$ \\
\hline $\begin{array}{l}\text { +Process_create }() \\
\text { +Process_delete }() \\
\text { +Process_update }() \\
\text { +Process_join }() \\
\text { +Process_suspend }() \\
\text { +Process_synchronize }()\end{array}$ \\
\hline
\end{tabular}

Figure 1. UML class diagram for process. ample of distributed computing system as shown in the Figure 2. In this various devices like laptops, handheld devices are connected using distributed approach and it allows resources sharing, file sharing, data sharing, etc. It requires low cost involvement and the processes are communicated through message passing technique. The facilities represented in the figure are Research_labs, Countrywide classroom, Universities, E-Commerce, EGovernance and Research Institutions and these facilities share the NKN network around the country. The NKN is capable of providing secure and reliable connectivity to data transmission from one device to the other device. The aim of this project is to connect all the universities, research Libraries, institutions and health care and agriculture institutions. The NKN supports transfer of data up to $1 \mathrm{Gbps}$ that can be further enhanced up to $20 \mathrm{Gbps}$ in future.

\subsection{Static Step Topology}

The $\mathrm{N}$ numbers of devices [14] are connected under distributed environment by varying the steps. One device acts as a server and other systems are connected sharing the facilities like libraries, institutions, research institutions, laboratories, countrywide classroom and sharing resource with each other. The global sharing of facilities provided by NKN under static step topology is shown below in Figure 3. The figure consists of three steps which consist of the seven devices and these devices are dedicated to NKN server, Virtual Library, Research Institutions, Research Labs, Sharing of Computing Resources, E-Governance and Country wide class room. However the other facilities may be extended by the use of the step topology. Since the complexity of step topology is of linear order, therefore, it is very easy to extend the facilities for $\mathrm{N}$ number of devices.

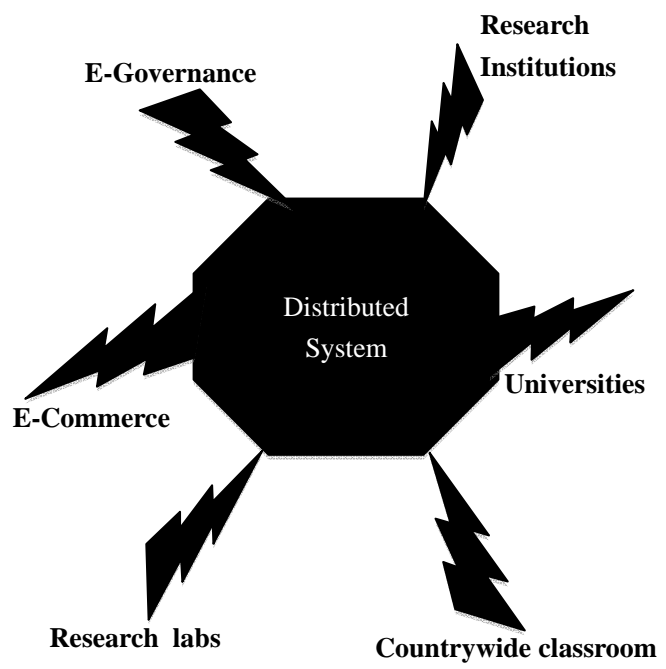

Figure 2. Distributed NKN network. 


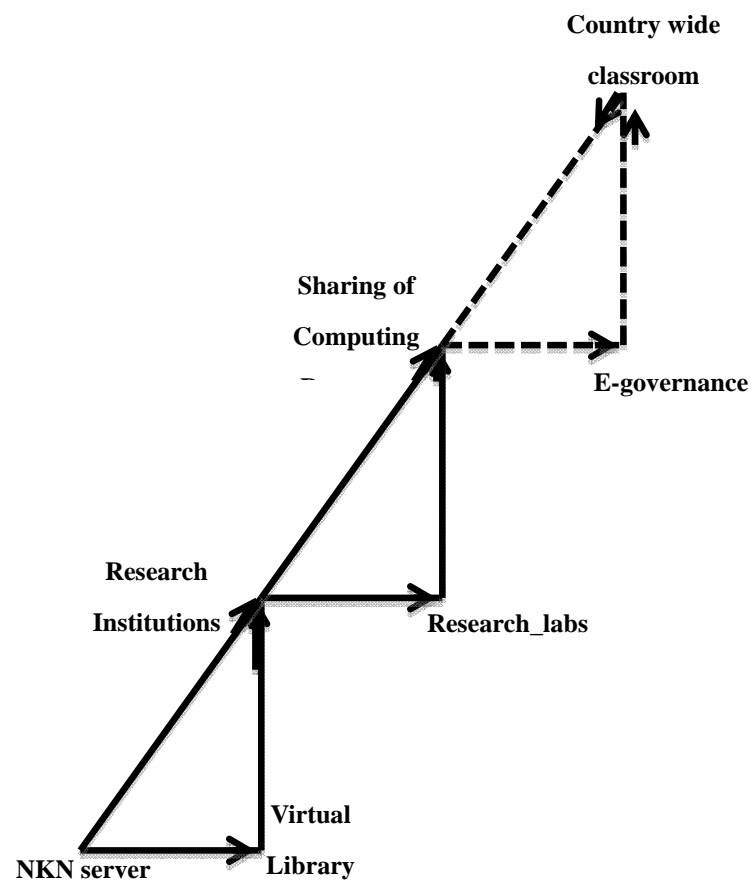

Figure 3. A static step topology.

\subsection{Resource Allocations}

The sharing of resources like audio file sharing, video file sharing, sharing of services connected under NKN are represented in the form of step topology. When the numbers of systems are connected around the globe then under distributed computing system they can share the resources and facilities, which are attached under NKN. The category of facilities may be file sharing, data sharing, videos sharing, audio sharing, virtual library facilities sharing, countrywide classroom facilities, research labs and institutions connectivity etc. In this system, if one wants to execute the task by taking the devices, which are allocated, at a very far distance then by remote execution, the task can be executed on these devices. This is called as the remote access of the devices and sharing of the resources of these devices.

\subsection{Process Migration}

Transmitting the process from one system to another computer system is called as process migration by allocating the processors for execution of tasks or sharing of resources under distributed environment. The inconsistency arises if the data from one computer system wants to migrate to other by using write back or write through cache policy. The write update and write invalidate policies are used for execution of tasks and it is controlled by message passing technique.

\section{UML Modeling for Process Execution}

For execution of processes and allocation of resources connected using step topology over NKN network, a general UML class model is proposed in Figure 4 which shows the static behavior of the problem in which attributes and operations are grouped together to form a class. The figure represents the class diagram for execution of tasks and sharing of resources, the processes which are resides on NKN server attached with step topology are executed and shared by the user. Various facilities are provided to the user connect to the NKN server. The UML class diagram for execution of tasks is shown below.

The different facilities are aggregated to form a class Facilities which is attached to the NKN Server class controlled by Administration. The different devices are attached through Step_Topology class on LAN by the use of L3_Switch. The users are attached on the devices through step topology and they can access the facilities through NKN Server and different facilities are provided by the Service_Provider class.

\section{Experimental Results}

Let us compute the performance of the sharing of data on NKN server. The variable $T^{*} T h$ and $R^{*} T h$ represent packet transmitted and received throughput and $\mathrm{T}^{*} \mathrm{Vol}$ and $\mathrm{R}^{*} \mathrm{Vol}$ represent total data volume transmitted and received, respectively. The devices used by the users are called as the traffic generator while the NKN server is the absorber. The Table 1 shows the relative network performance by showing transmitted, received throughput and volume, respectively. The similar results are depicted in the form of graphs in Figures 5 and $\mathbf{6}$.

\section{Conclusions and Future Scope of Work}

From the above it is concluded that the Unified Modeling

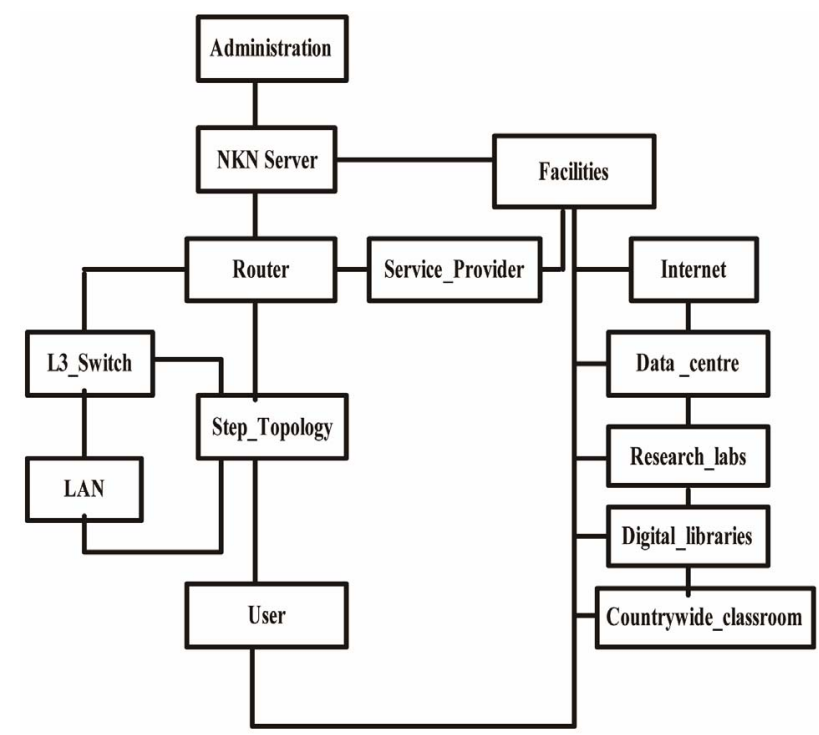

Figure 4. A UML diagram for process execution. 


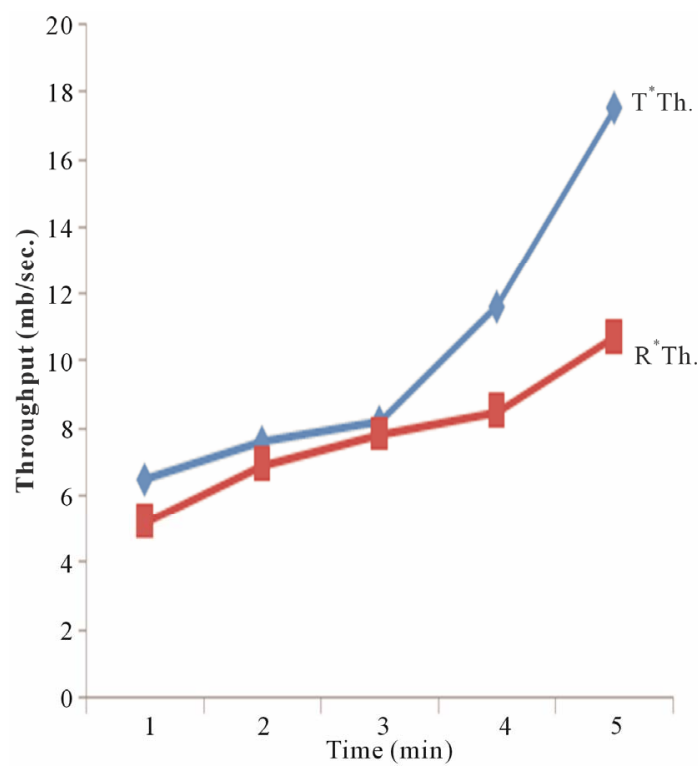

Figure 5. Transmitted and received throughput.

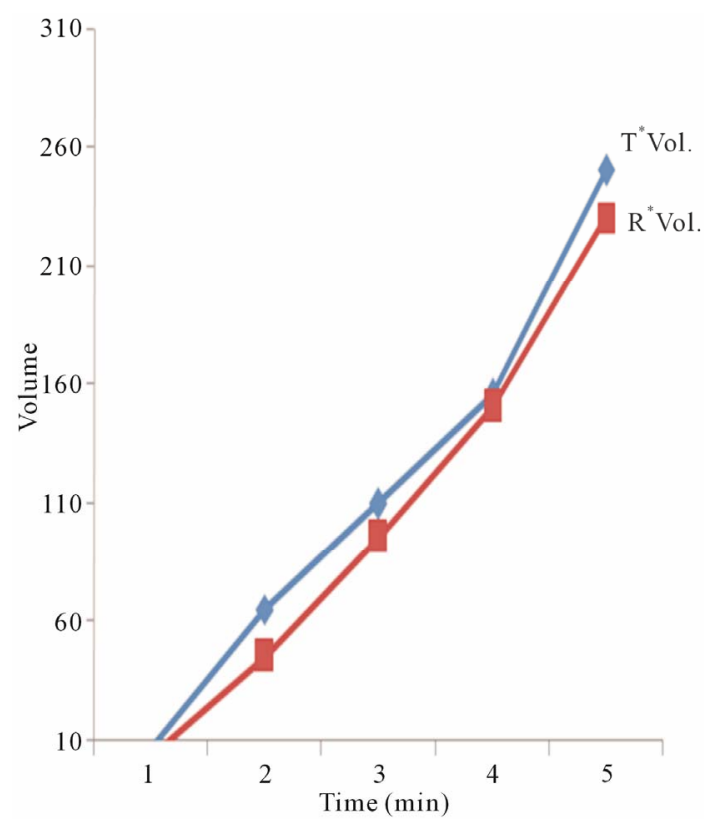

Figure 6. Transmitted and received volume of data.

Table 1. Network performance of data sharing on step topology.

\begin{tabular}{ccccc}
\hline Time (min.) & \multicolumn{2}{c}{ Sender } & \multicolumn{2}{c}{ Receiver } \\
\hline & $\mathrm{T}^{*}$ Th. (mb/sec.) & $\mathrm{T}^{*}$ Vol. (mb) $\mathrm{R}^{*}$ Th. (mb/sec.) $\mathrm{R}^{*}$ Vol. (mb) \\
\hline 1 & 6.54 & 7.24 & 5.23 & 3.25 \\
2 & 7.65 & 65.25 & 6.95 & 45.24 \\
3 & 8.25 & 110.25 & 7.85 & 95.64 \\
4 & 11.65 & 155.94 & 8.54 & 150.65 \\
5 & 17.54 & 250.78 & 10.75 & 230.75 \\
\hline
\end{tabular}

Language (UML) is used to a model for the execution of processes and allocation of resources in the static step topology connected under distributed environment. The performance for sharing of the resources for NKN is depicted in the form of table and graphs which show the utilization of the NKN server for sending and transmitting of the bytes of data. The present work can be extended for the route tracing using routing algorithms for selecting best path for allocations and sharing of resources. The deadlock detection and network failure in step topology are the future scope of work.

\section{REFERENCES}

[1] A. S. Tanenbaum, “Computer Networks,” Prentice Hall, Upper Saddle River, 2010.

[2] B. A. Frouzen, "Data Communications and Networks," Tata McGraw Hill, Noida, 2006.

[3] A. Siberschatz and P. B. Galvin, "Operating Systems Concepts," 5th Edition, John Wiley and Sons, Inc., Hoboken, 2008.

[4] S. Pllana and T. Fahringer, "On Customizing the UML for Modeling Performance Oriented Applications," The Unified Modeling Language, Springer-Verlag, Dresden, Germany, 2002.

[5] S. Pllana and T. Fahringer, "UML Based Modeling of Performance Oriented Parallel and Distributed Applications," Winter Simulation Conference, 8-11 December 2002. doi:10.1109/WSC.2002.1172922

[6] G. Booch, J. Rambaugh and I. Jacobson, "The Unified Modeling Language,” User Guide Addison Wesley, Raeding, 1999.

[7] G. Booch, "Object-Oriented Analysis and Design with Applications," Second Edition, Addison Wesley, 1994.

[8] OMG, "Unified Modeling Language Specification,” 2013. http://www.omg.org

[9] V. Saxena and D. Arora, "UML Modeling of a Protocol for Establishing Mutual Exclusion in Distributed Computer System,” International Journal of Computer Science and Network Security, Vol. 8, No. 6, 2008, pp. 227 235.

[10] V. Saxena and D. Arora, "Performance Evaluation for Object-oriented Software Systems," SIGSOFT Software Engineering Notes, Vol. 34, No. 2, 2009, pp. 1-5.

[11] 2011. www.nkn.in

[12] C. A. Gloria, P. O. Olayinola and O. Comfort, "Countering Traditional Learning and E-learning Methods in Higher Distance Education: Assessing Learness Preference," 4th International Conference on Distance Education (ICDLE), San Juan, 3-5 October 2010, pp. 187-190.

[13] N. Zhang and H. Bao, "Research on the Computer Technology for E-Learning in Higher Education," International Conference on E-Education, E-Business, E-Management and E-Learning, Sanya, 22-24 January 2010, pp. 295-298.

[14] T. Zaidi and V. Saxena, "Step Topology for Static Inter- 
connection of Computer Systems under Distributed Environment," 3rd World Conference on Information Technology, Bercelona, 14-17 November 2012, p. 32
[15] T. Zaidi and V. Saxena, "National knowledge Network versus Information Communication Technology," IT Floor, Vol. 2. No. 2, 2012, pp. 1-5. 